\title{
Análisis de los diferentes tipos de perfiles de los menores infractores
}

\author{
VICENT SISTERNES TERCERO \\ vternestercero@gmail.com \\ KEREN CUeRVo GómeZ \\ cuervo@psi.uji.es
}

\section{Resumen}

Gran cantidad de jóvenes cometen o se ven involucrados en algún tipo de delito durante su adolescencia. Este estudio pretende analizar los perfiles de los menores infractores desde las variables del género, la edad, la nacionalidad y el tipo de delito, centrándose también en la reincidencia de dichos menores.

Los participantes de este estudio son 395 menores del Juzgado de Castellón, de entre 14 y 18 años, y en su mayoría de género masculino. Se ha realizado un análisis de los expedientes de los menores del Juzgado de Menores de Castellón, analizando los números de expedientes de cada uno, la tipología de los delitos, los datos demográficos, etc.

Los resultados obtenidos proporcionan la edad a la que los menores cometen más delitos, el género predominante, el tipo de delito que suelen cometer, así como las nacionalidad que más delitos comete, y la reincidencia que existe en cada una de las variables.

Palabras clave: menores infractores, menores reincidentes, delincuencia juvenil, delincuencia en menores.

\section{Abstract}

Lots of young people commit or are involved in some type of offence during their adolescence. This study aims to analyze the profiles of juvenile offenders since the variables of gender, age, nationality and the type of crime, focusing also on the recidivism of such minors.

The participants in this study are 395 minors of the Juvenile of Castellón, between 14 and 18 years old, and mostly male. The criminal files of the minors have been analysed in order to check numbers of proceedings of each minor, the typology of crimes, demographics, etc.

Results show which are the common aspects on the juvenile related to gender, type of crime that often commit, as well as the nationality that more crimes comment, and recidivism that exists in each one of the variables.

Keywords: juvenile offenders' recidivist, juvenile, juvenile delinquency, delinquency in minors. 


\section{Introducción}

Es de gran relevancia para la sociedad actual, tener conciencia de la importancia que tienen los diferentes delitos cometidos por los adolescentes. Comparando diferentes estudios sobre la delincuencia en menores, se observa que un gran número de jóvenes se involucra en algún tipo de delito durante la adolescencia, sin embargo en la mayoría de casos solo cometen un delito de forma puntual (Capdevila, Ferrer y Luque, 2005). En otros países, como Reino Unido en el $80 \%$ (Jennings, 2002) y Australia el $70 \%$ de los jóvenes (Cain, 2000; Coumarelos, 1994), observamos que también se cumple esta tendencia.

Centrando el estudio en una variable importante para el perfil de los menores, como es el género, podemos comprobar que la violencia de las chicas en el ámbito familiar ha sufrido un incremento en los últimos tiempos, llegando a duplicar el porcentaje de los chicos. Las jóvenes que han sido denunciadas por los padres tienen una reincidencia violenta familiar muy elevada, del 33,3\% ante el 15,2 \% de reincidencia que presentan los chicos (Berga, 2005). En otros estudios también observamos que la población infractora es predominantemente masculina con un 87,3 frente al $12,7 \%$ de mujeres (Capdevila, Ferrer y Luque, 2005) coincidiendo con los datos estadísticos de la Dirección General.

Otros estudios muestran que la mayor diferencia derivada del género se encuentra en la conducta delictiva violenta, más grande en los hombres que en las mujeres. Otras diferencias que nombra se refieren al tiempo que se tarda en reincidir: si la chica reincide, lo hace de seguida, sino, no reinciden, mientras que el chico mantiene un periodo de riesgo más amplio. La chica también se inicia en la carrera delictiva un poco más tarde que el chico y la abandona antes (Rechea, 1999). Por otro lado, hay estudios que concluyen que el género explica la reincidencia pero la explica poco. Los hombres son más reincidentes que las mujeres, pero no en todos los programas aplicados (Capdevila et al., 2005).

La relación entre la edad y la criminalidad ha sido objeto de un considerable número de estudios, e incluso autores como Smith (1995) sugieren que, probablemente, la variable que mejor explica la delincuencia sea la edad. Si esto fuera así, parece que la mejor estrategia para reducir la incidencia de la criminalidad perpetrada por menores es sencillamente esperar a que se hagan mayores. (San Juan y Ocáriz, 2009). Varios estudios demuestran la tendencia de los jóvenes a realizar algún tipo de actividad delictiva alcanzando su máximo nivel a los 17 años y a partir de este punto la conducta se va normalizando, hasta llegar alrededor de los 18 años con una total normalidad, (Rechea y Fernández, 2006). Este aspecto se podría explicar por la llamada «curva de edad» que explica que la delincuencia suele declinar cuando la persona alcanza sus primeros años de madurez, al final del segundo decenio de la vida, por lo tanto cuando los menores van llegando al final de su adolescencia disminuye su rebeldía y se hace cada vez más social (Laub y Sampson, 2001). Estos autores ofrecen las siguientes razones: El desistimiento es el producto de la maduración, es decir cambios físicos y mentales, o bien el producto de cambios de identidad que acompañan a la edad, como consecuencia de cambios cognitivos, fisiológicos o sociológicos. La Teoría de Moffitt (1993), que explica que existen dos tipos de delincuentes, unos que empiezan pronto y tienen una larga carrera delictiva, y otros que empiezan tarde y solo transitoriamente son delincuentes. El delito declina naturalmente con la edad, ya que es una conducta habitual entre los adolescentes, y termina cuando uno se convierte en adulto (Gottfredson y Hirschi, 1990). El descenso en el delito se atribuye a la formación de controles sociales y formales o vínculos con los demás, tanto en la esfera personal como en el ámbito social. Según las teorías racionales, el desistimiento del delito es el producto de la decisión más racional que toman las personas cuando, generalmente, alcanzan la etapa adulta. 
También es de gran importancia conocer la nacionalidad de los menores delincuentes, ya que así podremos saber si influye de forma directa la nacionalidad de estos. La población extranjera en contacto con la justicia de menores ha ido creciendo, como lo ha hecho en todos los ámbitos de la vida social. La inmigración ha sido un fenómeno en alza muy acusado en los últimos años y de contrastado impacto sobre la estructura demográfica y social de nuestro país. Los extranjeros son más reincidentes que los españoles, si bien con una significación baja. De hecho, solamente lo son en los programas de mediación, es fácil explicarlo a partir de los problemas de lengua o diferencias conceptuales y culturales en las que el concepto de responsabilidad y reparación puede ser distinto al nuestro. Los extranjeros reinciden antes y lo hacen un número superior de veces (Capdevila et al., 2005).

Por otra parte varios estudios demuestran que la mayoría de delitos se realizan contra la propiedad, en robos y robo con violencia y robo con fuerza (Capdevila et al., 2005; Graña et al., 2006; Garrido, 2009). Tras estudiar diferentes resultados de otros estudios, se comprueba que el hecho cometido mayoritariamente en los menores estudiados han sido delitos (81.4 $\%$ ), siendo el delitos más frecuente contra la propiedad con un $65.8 \%$ y contra las personas un $22.1 \%$, por lo tanto según estos estudios parece ser que los delitos cometidos contra la propiedad son los que resultan más predictores de la reincidencia (Capdevila, 2005).

\section{Objetivo}

El objetivo general será explicar el perfil de los menores de la muestra, separándolos en reincidentes y no reincidentes, teniendo en cuenta el periodo de seguimiento que ha realizado el equipo técnico del Juzgado de Castellón, siendo un total de 24 meses. Donde se determinará el porcentaje de menores reincidentes, y el de no reincidentes, y explicar las diferencias de perfiles entre estos dos grupos, estudiando variables como: el sexo, la edad, la nacionalidad y el tipo de delito cometido.

\section{Método}

\section{Participantes}

Los participantes de este estudio son 395 menores del Juzgado de Castellón. El rango de edad de los jóvenes es de 14,03 a 18,10 años. El sexo es mayoritariamente masculino, siendo la proporción de 321 chicos que forman el $81,3 \%$ de los participantes, y 74 chicas que forman el $18,7 \%$ restante.

\section{Procedimiento}

Los datos de este estudio se obtienen a partir del análisis de los expedientes de los menores en el Juzgado de Menores de Castellón. Se analizaron el número de expedientes de cada menor, la tipología de los delitos cometidos, la medida propuesta por el equipo técnico, los datos demográficos y otros aspectos relacionados. 


\section{Resultados}

Los resultados obtenidos tras analizar los expedientes de la muestra de menores, proporciona tal información como que el total de sujetos de la muestra es de 395 menores. Como podemos apreciar en la Gráfica 1 el $15 \%$, que serían 60 menores, serían los reincidentes, quedando como no reincidentes el $85 \%$ del total de la muestra, siendo los 335 menores restantes.

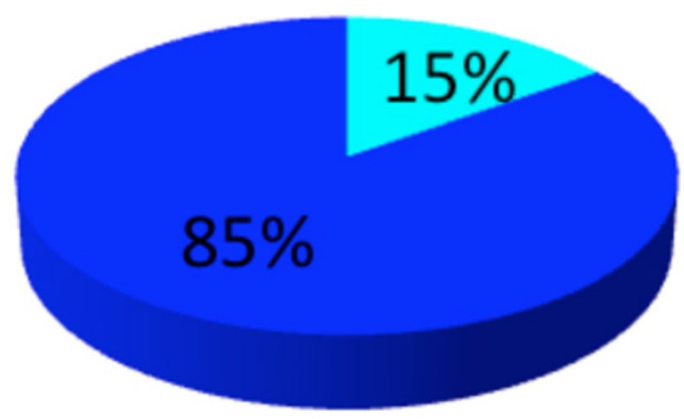

\section{- NO REINCIDENTES}

$\mathrm{N}: 335$

Gráfica 1. Menores reincidentes y no reincidentes

A continuación se presentan los resultados obtenidos que muestran las diferencias de perfiles en los menores reincidentes y los no reincidentes en relación con las siguientes variables:

- Sexo

- Edad

- Nacionalidad

- Tipo de delito

- Sexo

En relación al sexo de los menores, los resultados obtenidos demuestran que hay una mayoría de menores delincuentes de género masculino con un $81 \%$, que serían 321 sujetos, mientras que los delincuentes de género femenino corresponden solo al $19 \%$ de la muestra, siendo mucho menor el número de sujetos con 74 menores (Gráfica 2). 


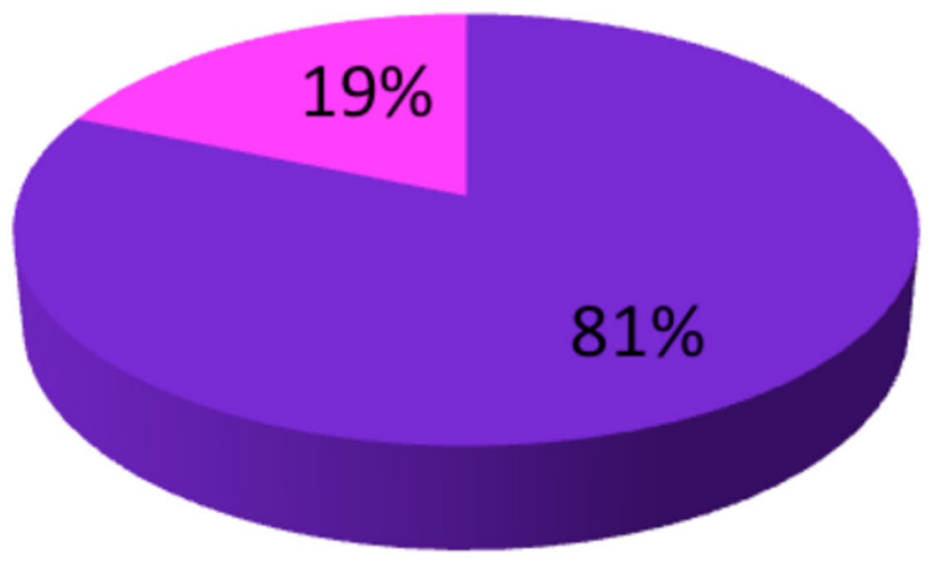

\section{- $\mathrm{CHICO}=\mathrm{CHICA}$}

Gráfica 2. Delincuencia por género

Como podemos apreciar en la siguiente gráfica (Gráfica 3), la diferencia entre género es bastante elevada, separando la muestra en reincidentes y no reincidentes. Dentro de los menores reincidentes se aprecia que el $85 \%$ son del género masculino, mientras que el género femenino tendría un $15 \%$. También es notable la diferencia dentro de los no reincidentes, con un $80.60 \%$ del género masculino, frente a un $19.40 \%$ del femenino. Por lo tanto es bastante mayor el número de menores del género masculino, tanto en reincidentes como en no reincidentes.

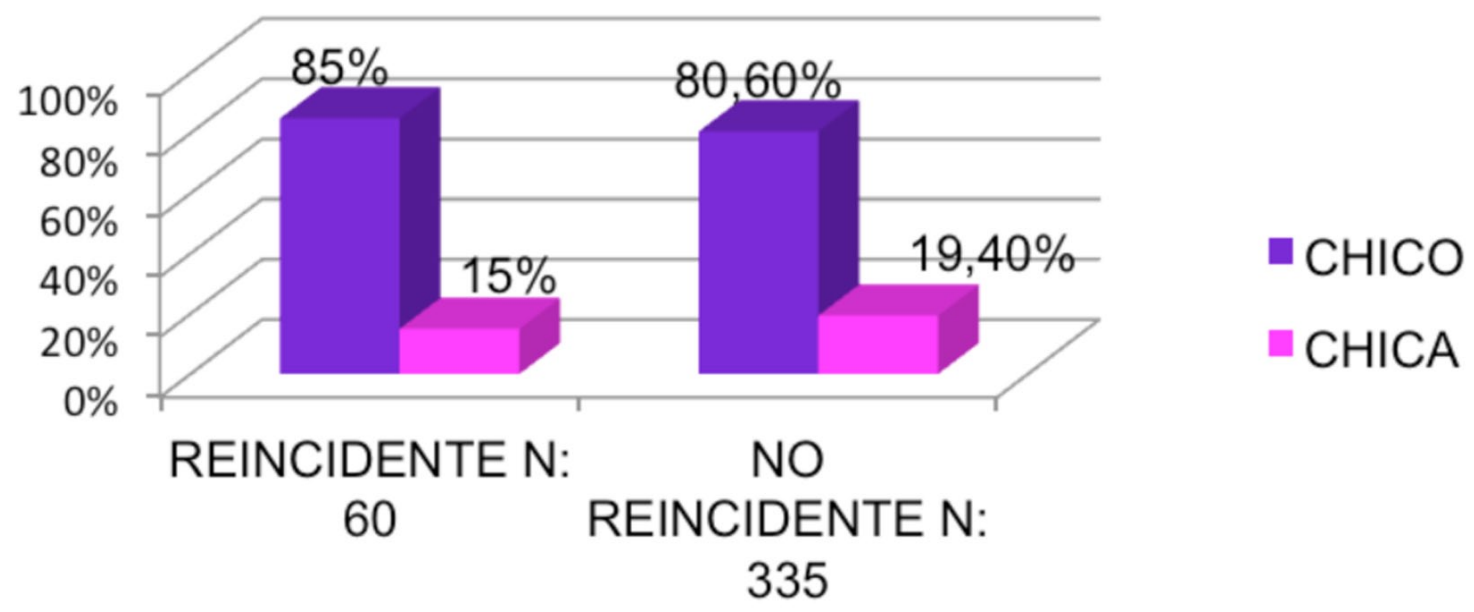

Gráfica 3. Diferencia reincidente-no reincidente

\section{Edad}

Por lo que se refiere a la edad, los menores son más reincidentes cuando son más jóvenes, es decir cuando se van acercando a la mayoría de edad, suelen reincidir en menor cantidad.

Dentro de los reincidentes, el porcentaje de 14 a 15,99 años y de 16 a 18 años es muy similar, con un $51,70 \%$ y un $48,30 \%$ respectivamente. Sin embargo, en los no reincidentes 
hay una gran diferencia, teniendo un $31 \%$ los menores entre 14 y 15,99 años, y un $69 \%$, los de 16 a 18 años. Lo que apoyaría varias teorías que defienden que los menores a medida que se van acercando a la mayoría de edad disminuyen su vida delictiva, ya que se va terminando su adolescencia (Gráfica 4).

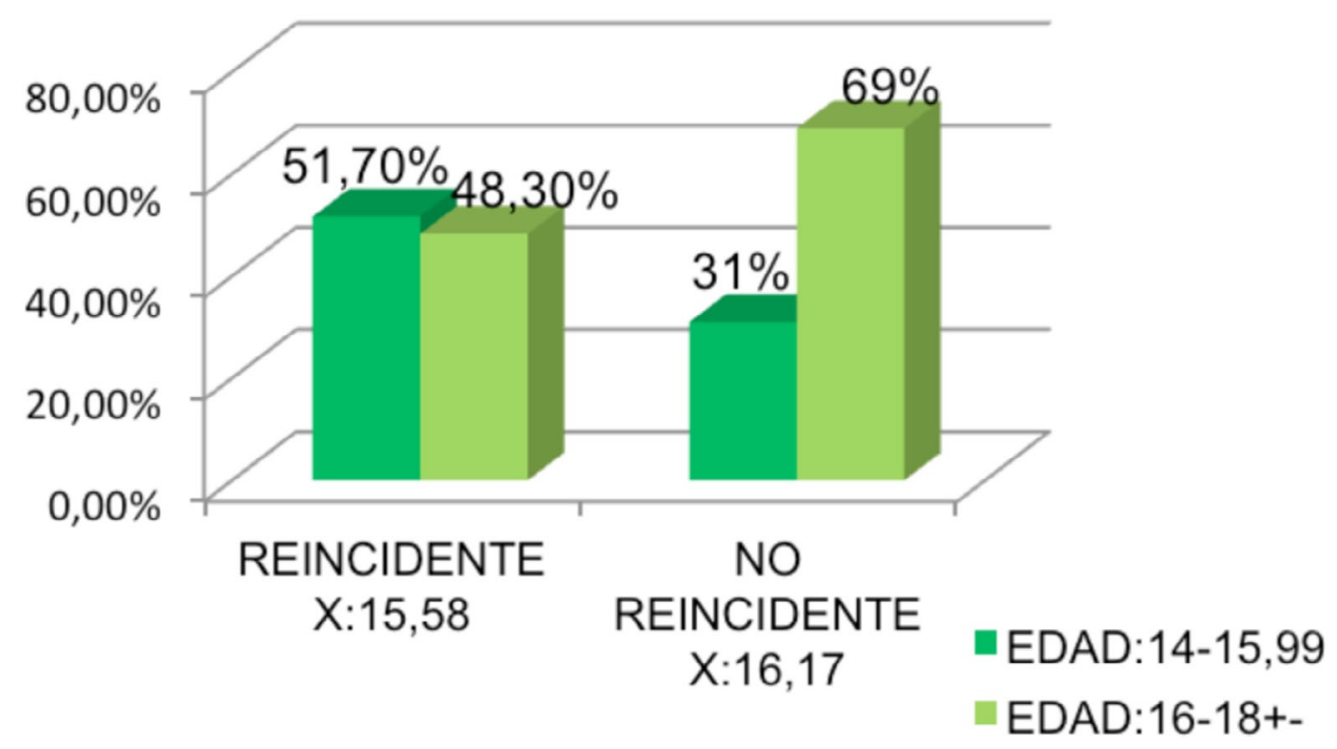

Gráfica 4. Reincidencia por edades

\section{Nacionalidad}

Por lo que se refiere a la nacionalidad, evidentemente la mayoría de delincuentes del Juzgado de Castellón son españoles, ya que están en su país de origen. Como se puede comprobar en la gráfica los españoles ascienden a un $77 \%$, siendo 303 menores, sin embargo, solo un $23 \%$, o lo que es lo mismo 92 sujetos, de los delitos en menores corresponden a delitos de extranjeros.

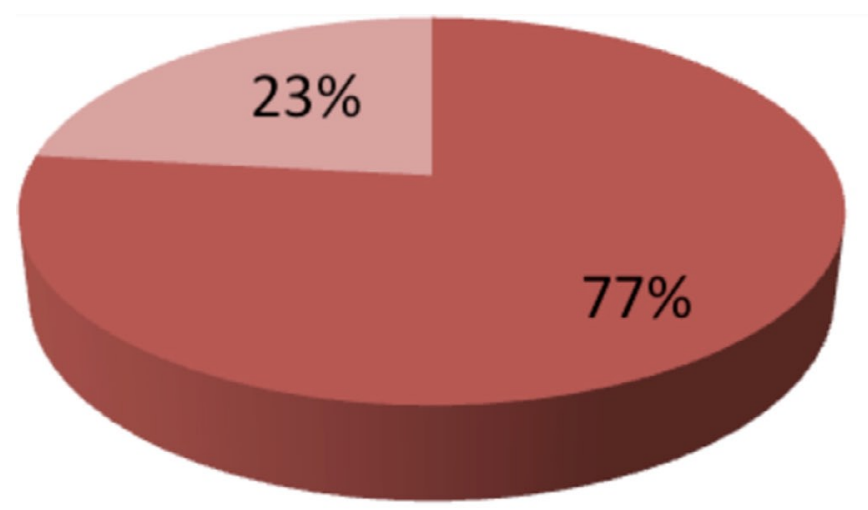

- ESPAÑOL

- EXTRANJERO

Gráfica 5. Delincuentes españoles y extranjeros 
Sin embargo, aunque la mayoría de los delitos son cometidos por españoles, son los extranjeros los que reinciden en mayor medida con un $17,4 \%$, frente al $14,5 \%$ de los españoles. Dentro de los extranjeros, los sudamericanos son los que tienen el mayor porcentaje de reincidencia con un $22,6 \%$, seguidos por los Países árabes con un $16 \%$, los españoles con un $14,5 \%$ y los rumanos con un $13,9 \%$ (Tabla 1 y 2 ) (Gráfica 6 ).

Tabla 1

Reincidencia por nacionalidad

\begin{tabular}{lccc}
\hline & REINCIDENTES & NO REINCIDENTES & TOTAL \\
\hline NACIONALIDAD & & & \\
Español & $44(14,5 \%)$ & $259(85,5 \%)$ & $303(76,7 \%)$ \\
Extranjero & $16(17,4 \%)$ & $76(82,6 \%)$ & $92(23,3 \%)$ \\
\hline
\end{tabular}

Tabla 2

Reincidencia por nacionalidades

\begin{tabular}{lccc}
\hline NAC. POR PAISES & REINCIDENTES & NO REINCIDENTES & TOTAL \\
\hline Español & $44(14,5 \%)$ & $259(85,5 \%)$ & $303(76,7 \%)$ \\
Rumano & $5(13,9 \%)$ & $31(86,1 \%)$ & $36(9,1 \%)$ \\
Sudamericano & $7(22,6 \%)$ & $24(77,4 \%)$ & $31(7,8 \%)$ \\
Países Árabes & $4(16,0 \%)$ & $21(84,0 \%)$ & $25(6,3 \%)$ \\
\hline
\end{tabular}

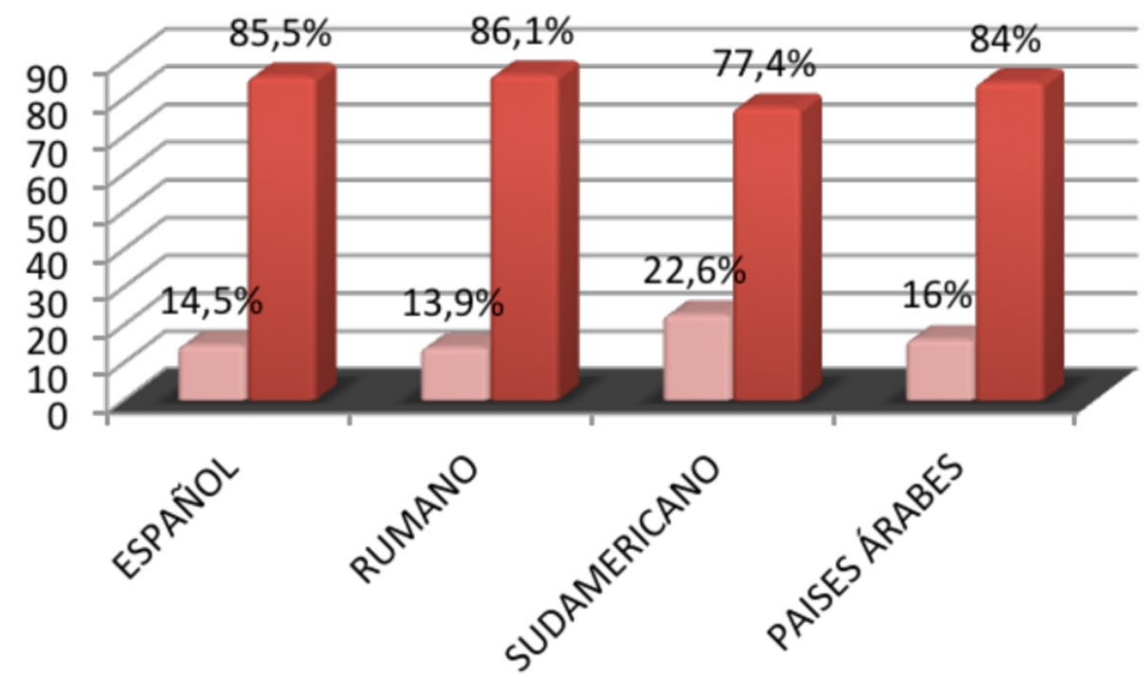

\author{
$\square$ REINCIDENTE \\ $\square$ NO REINCIDENTE
}

Gráfica 6. Delitos por nacionalidad 
Tipo de delito

Por lo que se refiere al tipo de delitos, hay bastante igualdad entre los delitos contra la persona $(51,4 \%)$ y contra la propiedad $(48,6 \%)$, aunque reinciden en mayor medida los menores que en la línea base del estudio han realizado delitos contra la propiedad con un 16.1 $\%$ frente a un $14.3 \%$ que son los que reinciden contra la persona (Gráficas 7 y 8 ).

- CONTRA PERSONA

CONTRA PROPIEDAD

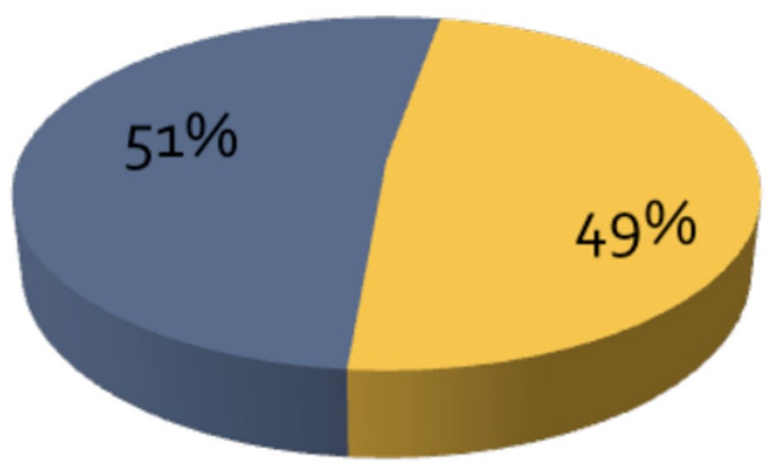

Gráfica 7. Delitos contra la persona-Delitos contra la propiedad

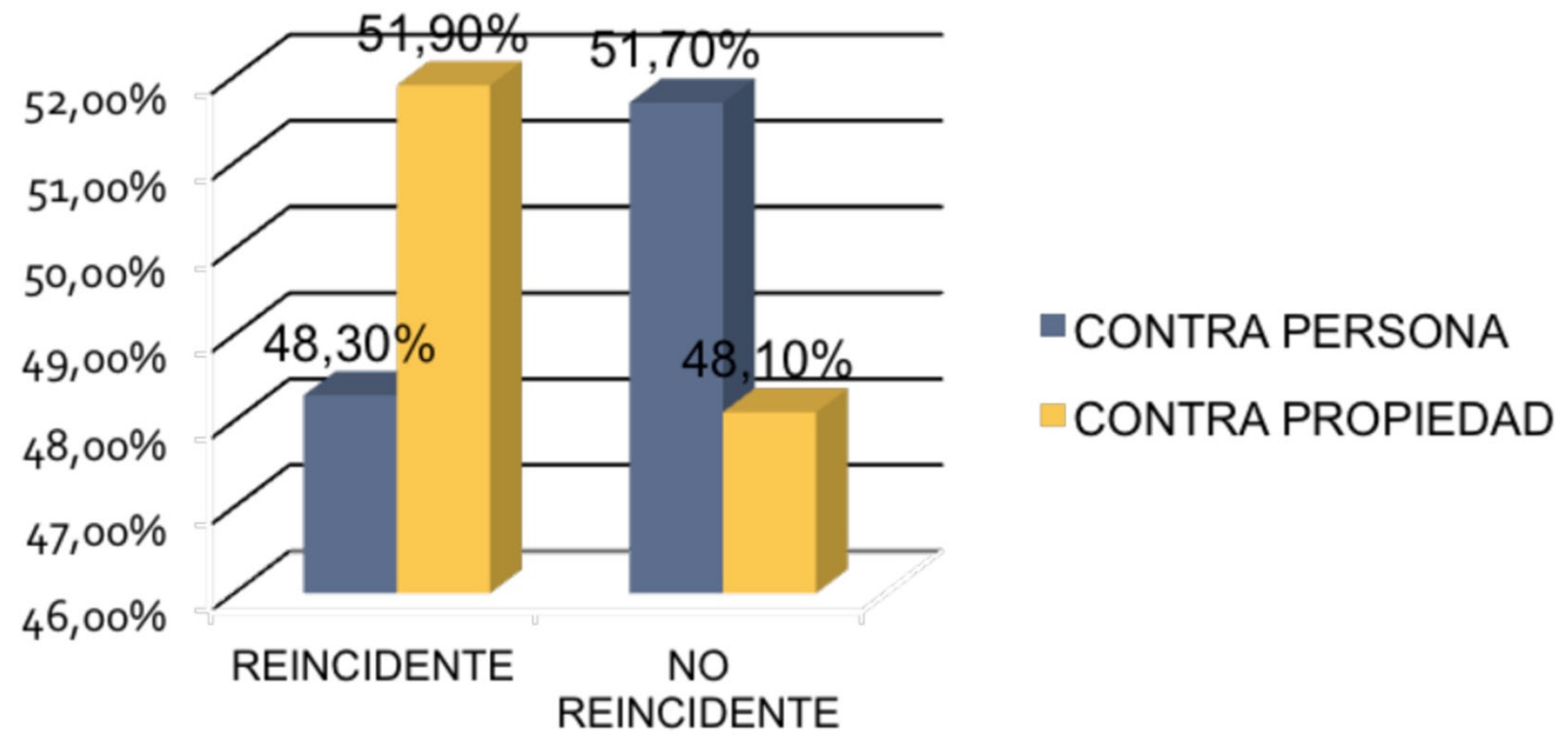

Gráfica 8. Delitos contra la propiedad y contra la persona en reincidentes-no reincidentes 


\section{Conclusión}

Tras realizar un estudio exhaustivo a la muestra de menores recopilada por el equipo de investigación del departamento de Psicología Evolutiva, Educativa, Social y Metodología de la Universidad Jaume I de Castellón, de 395 expedientes del Juzgado de Menores, se ha llegado a las siguientes conclusiones: Se puede apreciar que del total de la muestra el $85 \%$ de los menores no son reincidentes. Esto nos deja con un $15 \%$ de reincidentes que viene a ser 60 menores de la muestra total. Siempre comparando los dos grupos, es decir reincidentes y no reincidentes, se han utilizado diferentes variables que han proporcionado información de todos los menores en cuestión.

Tras utilizar la variable «sexo» ésta, indica que la mayoría de los menores de la muestra son del género masculino con un $81 \%$ de ellos, mientras que del género femenino tan solo hay un $19 \%$. Estos resultados son muy parejos con el estudio de Capdevila en 2005, que realizo con menores de Cataluña, obteniendo este un $87,3 \%$ del género masculino, y un 12,7 $\%$ del género femenino. Al separarlos en reincidentes y no reincidentes observamos que dentro de los reincidentes los que más reinciden también son los menores masculinos con un 85 $\%$, frente a un $15 \%$ de los menores del género femenino.

Posteriormente tras utilizar la variable «edad», se puede comprobar la teoría de «la curva de la edad», y no es otra que a medida que los menores se van acercando a la mayoría de edad disminuye sus ganas de delinquir, según esta teoría, sería porque al menor en cuestión se le está terminando la adolescencia y por tanto poco a poco es menos rebelde y más social. Esto explicaría los resultados obtenidos, ya que dentro de los reincidentes está prácticamente igualado el porcentaje de reincidencia en las edades de 14 a 15,99 y de 16 a 18 años, mientras que en el grupo de no reincidentes, mientras que el porcentaje de la edad entre $14 \mathrm{y}$ 15,99 es de $31 \%$, el del rango de edad de 16 a 18 años es de $69 \%$. Así que los menores de la muestra, a medida que se acercan a los 18 años disminuye notablemente si vida delictiva.

También se ha utilizado la variable «nacionalidad», de la cual pudimos extraer que como es normal hay mayor número de menores de nacionalidad española, con un $77 \%$, en el juzgado de Castellón, ya que están en su país de origen, frente a un $23 \%$ de extranjeros. Pero aun siendo así, los menores españoles no son los más reincidentes, estando en primer lugar los menores que provienen de diferentes países sudamericanos con un 22,6\% de reincidencia, seguidos de los menores provenientes de diferentes países árabes, con un $16 \%$, y en tercera posición se encuentran a los menores españoles con un $14,5 \%$, y en el último puesto estarían situados los menores de procedencia rumana con un $13,9 \%$ de reincidencia. En otro estudio que han utilizado la misma variable han obtenido resultados parecidos con la diferencia de que los menores de procedencia de países árabes se encuentran en el primer lugar de reincidencia con un $66,5 \%$ y en segundo lugar los menores procedentes de países del centro y del sur de América con un 15,5\% (Capdevila 2005).

Para terminar con las variables utilizadas, la última escogida es el «tipo de delito cometido», los resultados obtenidos demuestran que no existe prácticamente ninguna diferencia entre menores que han realizado un delito contra una persona y menores que lo han realizado contra una propiedad, aunque sí que existe una ligera diferencia entre el grupo de reincidentes donde los delitos contra la persona tiene un $51.90 \%$ frente un $48.10 \%$ en delitos contra la propiedad. Si lo comparamos con otros estudios similares, éstos sí demuestran bastante diferencia entre los delitos contra la propiedad con un $65.8 \%$ frente a un $22.1 \%$ en delitos cometidos contra la persona. Por lo tanto el delito cometido más frecuente es el cometido contra la propiedad (Capdevila 2005).

Por lo tanto tras los resultados obtenidos con la muestra de 395 menores, se concluye que, el perfil más común del menor reincidente tiene como más característicos los rasgos de 
género masculino, de edad entre 14 y 15,99 años y de nacionalidad Sudamericana, y también bastantes posibilidades de que el menor haya cometido el delito contra la propiedad.

\section{Referencias}

Berga, A. (2005). Adolescència femenina i risc social. Revista d'intervenció socioeducativa, 29, $61-76$.

Cain, M. (2000). An Analisys of Juvenile Recidivism. Recuperado de http//www.aic.gov.au/publications/rpp/ch2.pdf.

Capdevila, M., Ferrer, M. y Luque, E. (2005). La reincidencia en el delito en la justica de menores. Centre d'estudis Juridics i formació especialitzada. Barcelona: Generalitat de Catalunya.

Coumarelos, C. (1994). Juvenile offending: Predicting persistence and determining the costeffectiveness of interventions. Sydney: NSW Bureau of crime Statistics and research (BOSCAR).

Cuervo, K. (2011). Menores en Riesgo: Perfil y Predicción de la reincidencia delictiva. Tesis Doctoral. Universidad Jamue I.

Garrido, V. (2009). La predicción y la intervención con los menores infractores: Un estudio en Cantabria. (Estadísticas, instrumentos, protocolos y evaluación). Gobierno de Cantabria.

Graña, J. L., Garrido, V. y González, L. (2006). Reincidencia delictiva en menores infractores de la Comunidad de Madrid: Evaluación, características y propuestas de intervención. Madrid: Agencia para la Reeducación y Reinserción del Menor Infractor (ARRMI).

Gottfredson, Michael y Travis Hirschi. 1990. A General Theory of Crime. Stanford: Stanford University Press.

Jennings, D. (2002). One Year Juvenile Reconviction Rates. July 2000 cohort. UK. Home Office National Statistics: London.

Moffit, T. E. (1993). Adolescence-limited and life-course-persistent antisocial behavior: A developmental taxonomy. Psychology review, 100, 674-701.

Laub, J. y Sampson, J. (2001). Boys in Trouble and How They Age. Cambridge, Mass.: Harvard University Press.

Rechea, C. y Fernandez, E. (2006). Continuity and change in the spanish Juvenile Justice System. En J. Junger-tass, y S. Decker (eds.) Handbook of juvenile Justice (pp. 325-350). Holland: Kluwer/Springer.

San Juan, C. y Ocáriz, E. (2009). Evaluación de la intervención educativa y análisis de la reincidencia en la Justicia de Menores en la CAPV. Vitoria-Gasteiz. Servicio Central de publicaciones del Gobierno 